\title{
Signal Timing for an Isolated Intersection Based on the Two-Stage Fuzzy Logic Controller
}

\author{
Junjie Lu, Fuyang Chen ${ }^{*}$ and Lin Ma \\ College of Automation Engineering, Nanjing University of Aeronautics and Astronautics, Nanjing, China \\ ${ }^{*}$ Corresponding author
}

\begin{abstract}
This paper proposes a two-stage fuzzy logic controller for signal timing of an isolated intersection. In the first stage, the main phase is determined in advance, and then the controller determines the secondary phase according to the traffic flow and the queue length. In addition, the membership function is designed to adapt to the changing traffic flow. In the second stage, traffic emergency degree is calculated in real time to adjust the green time. In this case, the traffic arrival rate can be indirectly considered. Finally, the average vehicle delay is used to compare the effectiveness of each signal timing scheme. The simulation result shows that the two-stage fuzzy controller proposed in this paper can achieve good control effect.
\end{abstract}

Keywords-fuzzy logic controller; signal timing; adaptive membership function; vehicle delay

\section{INTRODUCTION}

With the growing imbalance between traffic demand and transportation resources, intelligent signal timing has gained attention. Many of the traffic control methods are model based. L. Shen proposes a dynamic platoon dispersion model to predict the evolution of traffic flow, and further used to produce signal timing plans [1]. In [2], a delay-based traffic signal control algorithm is applied to deal with the excessive delays and achieve better fairness with respect to delay. Reference [3] develops a signal control algorithm that allows for vehicle paths and signal control to be jointly optimized based on advanced communication technology. These adaptive methods need to model vehicle behavior. However, it is difficult to accurately model the actual traffic behavior and optimization algorithms based on the model may be time consuming.

Besides the model-based methods, the fuzzy control algorithm which is a model-free method can describe the urban traffic system reasonably as well. A two-stage fuzzy logic controller is proposed in [4-5] and it is a traditional design method. In general, the first step is to determine the signal phase and then the second step is to determine the green time delay. Because the signal phase designed by the fuzzy controller is unordered, only the secondary phase sequence is designed in this paper and the main phase is determined in advance. [6-8] introduce a type-2 fuzzy logic control method. The membership function of the fuzzy logic controller is also fuzzy, so that it can better adapt to the time-varying traffic flow. However, configuring high-dimensional complex parameters of the controller is difficult. Azimirad [9] combines fuzzy logic controller with state space equation to optimally control traffic flows under both normal and exceptional traffic conditions. In this way, modern control theory can be applied but the modeling process simplifies a lot of conditions. Fuzzy neural network system is introduced to deal with the prediction problem in [10-11]. This system needs a lot of data for training to determine the fuzzy parameters. This structure is effective for a particular problem but difficult to promote. In this paper, the proposed controller is easy to optimize to adapt to different traffic conditions.

The remainder of this paper is organized as follows. Section II discusses the structure of the two-stage fuzzy logic controller. Section III proposes the adaptive membership function to describe the changing traffic volume. Section IV describes the details of the simulation to verify the effectiveness of the proposed controller.

\section{Two-StAge Fuzzy LOGIC CONTROLleR}

This paper researches an isolated four-way intersection with straight and left-turning vehicle movements. The four phases are shown in Figure I. Right-turning vehicle movement is not limited by the signal light.

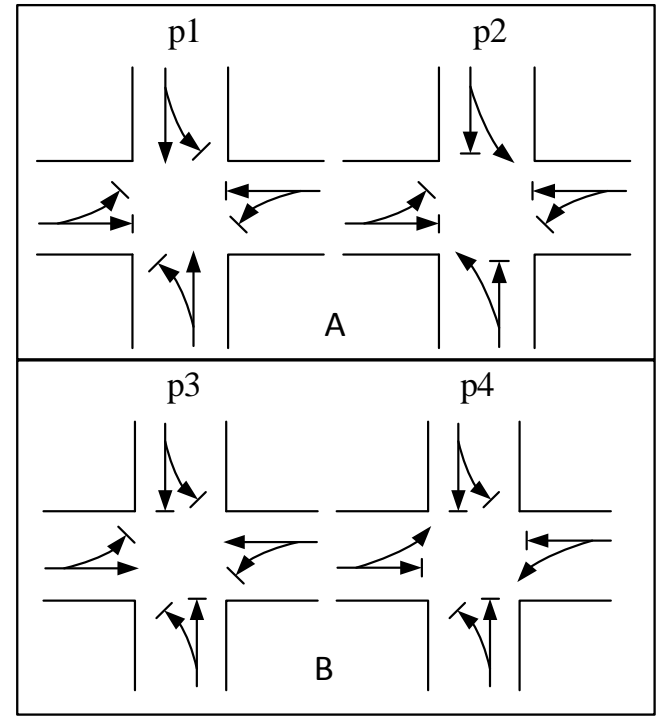

FIGURE I. FOUR-PHASE SIGNALIZED INTERSECTION

The fuzzy signal controller, including phase selection module and green time detection module, is different from traditional ones. Unlike disordered phase selection, each phase 
is guaranteed to be executed once in one cycle. Phase selection can't be entirely decided by the controller because the low flow phase may never be executed, however, the high flow phase is executed many times. We add some fixed order to deal with the problem. From Figure 1, the A process and the B process are executed alternately. The module determines which phase is executed first in the A process or in the B process. (p1 or p2; $\mathrm{p} 3$ or $\mathrm{p} 4)$.

The purpose of green time detection module is to determine whether to change the current phase. The input variables include queue vehicle number of current phase and average queue number of other phases. If the fuzzy controller directly determines the green time, vehicle arrival rate is ignored in this system and the result is not reasonable. Output is a decision variable. When the threshold is reached, the current phase is changed. In this case, the fuzzy controller is adaptive and indirectly considers the vehicle arrival rate.

\section{A. Phase Selection Module}

This module is used to determine whether the vehicles turn left or go straight. The east-west direction or the north-south direction is determined by the previous phase. There are two input variables and one output variables for the phase selection module. One input is the number of queue vehicles in this phase, defined as QL. Another is average vehicle arrival rate within 15 minutes, defined as AR. There is traffic flow of two directions in the same phase and we take the mean value of the two. The output variable is traffic urgency degree defined as U. The membership functions of $\mathrm{QL}, \mathrm{AR}$, and $\mathrm{U}$ are defined as follows:

The domain of AR is [0 0.25] and it represents the traffic flow per second. AR is divided into 5 fuzzy subsets \{Very Low, Low, Normal, High, Very High $\}$, for short $\{\mathrm{VS}, \mathrm{S}, \mathrm{A}, \mathrm{H}, \mathrm{VH}\}$; The domain of $U$ is [ 05$]$ and it is divided into 5 fuzzy subsets \{Very Light, Light, Normal, Heavy, Very Heavy\}, for short $\{\mathrm{VR}, \mathrm{R}, \mathrm{G}, \mathrm{F}, \mathrm{VF}\}$. The type of the membership function is triangle. The triangular membership function is conducive to our modification to adapt to the changing traffic conditions.

The fuzzy rules are shown in Table I.

TABLE I. FUZZY RULES OF THE PHASE SELECTION MODULE

\begin{tabular}{|c|c|c|c|c|c|}
\hline AR & VL & L & N & M & VM \\
\hline VS & VR & VR & R & G & F \\
\hline S & VR & R & G & G & F \\
\hline A & VR & R & G & F & VF \\
\hline H & R & G & F & F & VF \\
\hline VH & R & G & F & VF & VF \\
\hline
\end{tabular}

The element of the first row and first column is "VR" and it means if QL is VL and AR is VS, then U is VR. This rule can be described by natural language as follows: if there are very few queue vehicles and the arrival rate is very low, then the traffic urgency degree is very light. Fuzzy rules are not made at random and the weight of the two inputs is different. AR has a long-term impact on vehicle delays and QL has an immediate impact. So, the traffic urgency degree is mainly influenced by $\mathrm{QL}$, and AR plays a regulatory role.

\section{B. Green Time Detection Module}

There are two input variables and one output variables for the green time detection module. One input is the number of queue vehicles in this phase, defined as MQL. Another is average number of queue vehicles in other phases, defined as OQL. The output variable is a decision variable defined as J. It is fuzzed into a real value output between 0 and 1 . When the value exceeds a certain threshold, the green light signal switches to the red light phase, otherwise the green light signal will remain unchanged. In this paper, the threshold is 0.5 . The triangle membership functions of MQL, OQL, and $\mathrm{J}$ are defined as follows:

The domain of MQL is [0 30] and it is divided into 5 fuzzy subsets \{Very Few, Few, Normal, Many, A great many\}, for short $\{\mathrm{VS}, \mathrm{S}, \mathrm{A}, \mathrm{H}, \mathrm{VH}\}$. The domain of OQL is [0 30] and it is divided into 5 fuzzy subsets \{Very Few, Few, Normal, Many, A great many $\}$, for short $\{\mathrm{VL}, \mathrm{L}, \mathrm{N}, \mathrm{M}, \mathrm{VM}\}$. The domain of $\mathrm{U}$ is [0 1] and it is divided into 2 fuzzy subsets $\{$ Yes, No\}, for short $\{\mathrm{Y}, \mathrm{N}\}$.

The fuzzy rules are shown in Table II. The element of the first row and first column is "Y" and it means if OQL is VL and MQL is VS, then $\mathrm{J}$ is Y. This rule can be described by natural language as follows: if there are very few queue vehicles in current phase and very few queue vehicles in other phases, then the current phase can be switched. Even if there are congestion trends in other phases, we are still inclined to evacuate the current phase car flow.

\section{TABLE II. FUZZY RULES OF THE GREEN TIME DETECTION MODULE}

\begin{tabular}{|c|c|c|c|c|c|}
\hline OQL & VL & L & N & M & VM \\
\hline VS & Y & Y & Y & Y & Y \\
\hline S & N & N & N & Y & Y \\
\hline A & N & N & N & N & Y \\
\hline H & N & N & N & N & N \\
\hline VH & N & N & N & N & N \\
\hline
\end{tabular}

\section{The Control Algorithm}

It is assumed that the two phases in the same direction are paired. The first phase direction is initialized. $D_{g}$ is the traffic delay in the green phase, $D_{r}$ is the traffic delay in the red phase, $D_{A}$ is the average vehicle delay. The control algorithms include following steps:

Step 1. Choose the given direction; determine the phase according to the phase selection module of this direction.

Step 2. Set a minimum and maximum green time; delay the green time according to the green time detection module.

Step 3. Switch to the paired phase of this direction; next step is the same as step 2.

Step 4. Choose another direction; determine the phase according to the phase selection module of this direction. Refer to step 2 and step 3 to determine the green time of the third and fourth phases. 
Step 5. Calculate $D_{g}$ of this cycle and $D_{r}$ of previous cycle.

Step 6. Skip to step 1; if this cycle is the last cycle, calculate $D_{r}$ of this cycle separately; Get $D_{A}$ according to the vehicle flowrate.

\section{THE AdAPTIVE MEMBERSHIP FUNCTION}

Considering the changing traffic flow, a single membership function may have misleading effect on phase selection. An adaptive membership function needs to be constructed, a general membership function is defined as:

$$
u_{A}=\left\{\begin{array}{l}
\left(x-x_{i-1}\right) /\left(x_{i}-x_{i-1}\right), x \in\left(x_{i-1}, x_{i}\right) \\
\left(x_{i+1}-x\right) /\left(x_{i+1}-x_{i}\right), x \in\left(x_{i}, x_{i+1}\right) \\
0, \text { other }
\end{array}\right.
$$

The values of the three parameters $\left(x_{i-1}, X_{i}, X_{i+1}\right)$ can be adjusted to change the membership function according to the changing traffic flow. If traffic flow is decreasing, the new $X_{i-1}$ is:

$$
x_{i-1}^{\prime}=x_{i-1}-k \eta
$$

where $k$ is a ratio coefficient, $\eta$ is the increased traffic flow compared to the average traffic flow. $X_{i+1}, X_{i}$ don't change.

For example, the average traffic flow is $0.125 \mathrm{veh} / \mathrm{s}$, while current traffic flow is $0.2 \mathrm{veh} / \mathrm{s} . \eta$ is 0.3 , the membership function before and after the change is shown in Figure II. The dashed line represents the changed membership function.

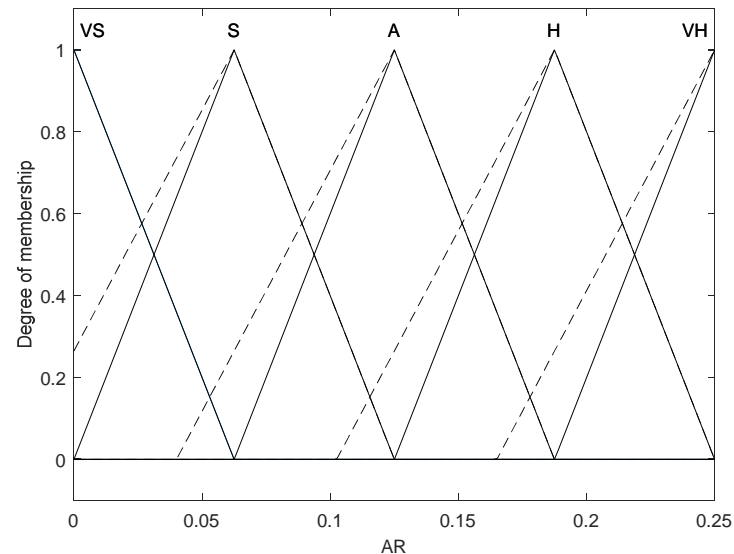

FIGURE II. MEMBERSHIP FUNCTION BEFORE AND AFTER THE CHANGE
We just made a conservative change, if necessary, $x_{i+1}$ also can be changed as:

$$
x_{i+1}{ }^{\prime}=x_{i+1}-k \eta
$$

If traffic flow is increasing, similarly, the new $x_{i-1}$ and $x_{i+1}$ are:

$$
x_{i-1}{ }^{\prime}=x_{i-1}+k \eta, \quad x_{i+1}{ }^{\prime}=x_{i+1}+k \eta
$$

The coefficients can be adjusted according to the actual situation.

The arrival rate of traffic flow plays an important role in vehicle queuing. The purpose of changing membership function is to avoid the condition that the arrival rate of traffic flow has small or great effect on fuzzy control under low or high flowrate situations.

\section{SIMULATION}

Data used in the simulation are collected from a single intersection in Huagang Road, Nanjing, Jiangsu Province, China. The simulation is done on MATLAB and vehicle delay is what we are concerned about. The saturated flow rate is $0.5 \mathrm{veh} / \mathrm{s}$ and the range of green time is $[15,51]$.

Figure III shows the control performance of fuzzy controller in this paper and traditional fuzzy controller [5]. The average vehicle delay is recalculated every cycle. Considering the vehicle arrival rate, we can see the traffic delay is obviously reduced compared to the traditional fuzzy controller. The control effect of the controller proposed in this paper is better than that of the traditional controller. The delay curve of the traditional controller is undulating because it can't adapt well to the time-varying traffic flow.

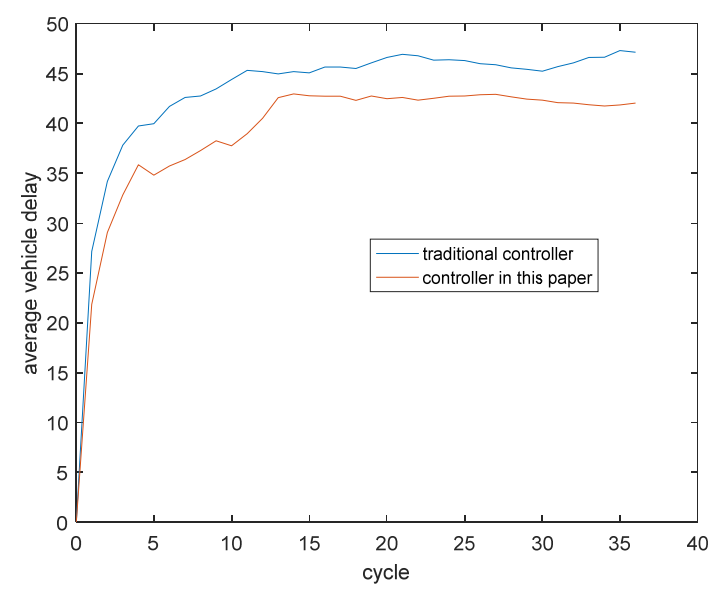

FIGURE III. TRAFFIC DELAY IN DIFFERENT CASES 


\section{CONCLUSION}

From the simulation results, it can be seen that the fuzzy controller proposed in this paper can effectively reduce the vehicle delay. The traditional fuzzy controller ignores the influence of the vehicle arrival rate, which leads to the poor control effect. The adaptive membership function of phase selection module is designed for a long-term control and the result shows the phase selection is also more accurate for a short term.

\section{ACKNOWLEDGMENT}

The project was financially supported by National Natural Science Foundation of China (61473146). The project funded by the Priority Academic Program Development of Jiangsu Higher Education Institutions.

\section{REFERENCES}

[1] Shen, Luou, et al. "Development of Dynamic Platoon Dispersion Models for Predictive Traffic Signal Control." IEEE Transactions on Intelligent Transportation Systems PP.99(2018):1-10.

[2] J. Wu, D. Ghosal, M. Zhang and C. N. Chuah, "Delay-Based Traffic Signal Control for Throughput Optimality and Fairness at an Isolated Intersection," in IEEE Transactions on Vehicular Technology, vol. 67, no. 2, pp. 896-909, Feb. 2018.

[3] Li, Zhuofei, L. Elefteriadou, and S. Ranka. "Signal control optimization for automated vehicles at isolated signalized intersections." Transportation Research Part C Emerging Technologies 49.49(2014):118.

[4] W. Yang, L. Zhang, Z. He and L. Zhuang, "Optimized two-stage fuzzy control for urban traffic signals at isolated intersection and Paramics simulation," 2012 15th International IEEE Conference on Intelligent Transportation Systems, Anchorage, AK, 2012, pp. 391-396.

[5] Yan, Ge. A two-stage fuzzy logic control method of traffic signal based on traffic urgency degree. Hindawi Publishing Corp. 2014.

[6] Y. Bi, X. Lu, D. Srinivasan, Z. Sun and Z. Sun, "Optimal Type-2 Fuzzy System For Arterial Traffic Signal Control," in IEEE Transactions on Intelligent Transportation Systems.

[7] Zhen Gao, FengHua Zhu and Hong Mo, "Traffic light time adjustment based on interval type-2 fuzzy sets," Proceedings 2014 International Conference on Informative and Cybernetics for Computational Social Systems (ICCSS), Qingdao, 2014, pp. 13-17.

[8] Zhang, Chi, et al. "Trajectory Tracking Control for Rotary Steerable Systems Using Interval Type-2 Fuzzy Logic and Reinforcement Learning." Journal of the Franklin Institute (2017).

[9] Azimirad, Ehsan, N. Pariz, and M. B. N. Sistani. "A Novel Fuzzy Model and Control of Single Intersection at Urban Traffic Network." IEEE Systems Journal 4.1(2010):107-111.

[10] Fu, Tian, and Z. Wang. "Based on Wavelet Analysis-Fuzzy Neural Network Real Time Traffic Flow Prediction." International Conference on Frontier Computing 2016:173-178.

[11] Aggarwal, Apoorva, A. Purwar, and S. Gulati. "An efficient technique to control road traffic using Fuzzy Neural Network System." International Conference on Reliability, INFOCOM Technologies and Optimization IEEE, 2015:1-6. 